\title{
Development of a FRET-based High-Throughput Screening System for the Discovery of Hsp90 Inhibitors
}

\author{
Sangmi Oh, ${ }^{\dagger}$ Yeonjin Ko, ${ }^{\dagger}$ Hanjae Lee, ${ }^{\dagger}$ Jonghoon Kim, ${ }^{\dagger}$ Young Sun Chung, ${ }^{\dagger}$ and Seung Bum Park ${ }^{\dagger},{ }^{,}{ }^{\star}$ \\ ${ }^{\dagger}$ Department of Chemistry and ${ }^{\S}$ Department of Biophysics and Chemical Biology, Seoul National University, \\ Seoul151-747, Korea.*E-mail: sbpark@snu.ac.kr \\ Department of Counseling, Korea Cyber University, Seoul 110-340, Korea \\ Received June 30, 2011, Accepted July 13, 2011
}

\begin{abstract}
A FRET-based high-throughput screening system was developed for the discovery of competitive smallmolecule Hsp90 inhibitors. The biarsenical fluorescein derivative FlAsH and dabcyl-conjugated Hsp90 inhibitor GM were employed as the FRET donor and quencher, respectively. The spatial proximity perturbation between FlAsH-labeled Hsp90N and GM-dabcyl upon treatment of a small molecule led to changes in the FRET-induced fluorescence, monitored in a high-throughput fashion.
\end{abstract}

Key Words : Hsp90, FlAsH, Fluorescent resonance energy transfer (FRET), High throughput screening (HTS), Benzopyran analogs

\section{Introduction}

Heat shock protein 90 (Hsp90) is one of the most abundant proteins in cells and associated with non-native structures and the maturation of many cellular proteins. Hsp90 is called as a molecular chaperone and modulates protein folding, intracellular arrangements and the proteolytic degradation of many client proteins to regulate cell growth and survival. ${ }^{1}$ Some of these client proteins play essential roles in promoting cancer cell growth and survival. ${ }^{1 \mathrm{~d}}$ In addition, Hsp90 is overexpressed in most cancer cells and critically regulates the stabilization of mutant proteins in genetically unstable cancer cells. Therefore, the specific inhibition of Hsp90 is one of the validated approaches for the treatment of various cancers. $^{2}$

Many reports have addressed specific small-molecule inhibitors of Hsp90, including geldanamycin (GM) ${ }^{3}$ and its analogs (17AAG and 17DMAG) ${ }^{4}$ radicicol, ${ }^{3 \mathrm{~b}} \mathrm{CNF}-2024^{5}$ and SNX-2112. ${ }^{6}$ In vitro and in vivo anticancer activity is induced by the competitive binding of these inhibitors at the ATP-binding pocket of Hsp90 N-terminal domain and subsequent ATP-dependent chaperone activity blockage. In addition, the identification of novel small-molecule Hsp90 inhibitors with improved pharmacokinetic properties is of great significance as a promising combination therapy for the cancer treatment. Although several Hsp90 inhibition assays have been reported including isothermal titration calorimetry $^{3 \mathrm{~b}}$ and radioisotope-based displacement using $\left[{ }^{3} \mathrm{H}\right]$-labeled $17-\mathrm{AAG}^{7}$ these methods are not fully compatible with the high-throughput screening (HTS) format because of their limitation in miniaturization and requirements of complex washing steps and hazardous radioisotopes. Fluorescence polarization has been widely employed for the development of HTS system. ${ }^{8}$ In addition, there are several other high-throughput Hsp90 assay systems such as surface plasmon resonance (SPR)-based binding assay, ${ }^{9}$ the coupled enzyme assay, ${ }^{10}$ and HTS assay based on refolding of firefly luciferase. ${ }^{11}$ However, these assay systems still failed to meet the full requirements of HTS.

In this manuscript, we describe the design and development of a novel FRET-based HTS assay system using the $N$ terminal domain of Hsp90 (Hsp90N) for the discovery of new molecular frameworks of competitive small-molecule Hsp90 inhibitors. FRET is a mechanism that demonstrates the energy transfer between donor and acceptor chromophores through non-radiative, dipole-dipole coupling with an inverse sixth power distance dependence. ${ }^{12}$ Therefore, FRET systems have been extensively used to quantify molecular dynamics or motions in biophysics and biochemistry, such as proteinprotein interactions and protein conformational changes. We envisioned the application of the FRET event between Hsp90N and GM, a known specific inhibitor of the Hsp90N ATP-binding domain, as a screening system for the discovery of novel competitive small-molecule inhibitors of Hsp90N for anticancer therapeutics.

\section{Results and Discussion}

The precise distance control between the acceptor and donor fluorophore is crucial for the development of an accurate FRET system. Therefore, the random chemical modification of Hsp90N with organic fluorophores is not suitable. One of the typical approaches for the site-specific labeling of fluorophore is the genetic incorporation of fluorescent proteins, such as green fluorescent protein (GFP). ${ }^{13}$ However, GFP might perturb the function of Hsp90 because of its large size (27 kDa). In contrast, 4',5'-bis(1,3,2dithioarsolan-2-yl)fluorescein (F1AsH), developed by the Tsien group, can be introduced to the proteins of interest in a site-specific manner. FlAsH is a small organic fluorophore, which is nonfluorescent until it is bound to the tetracysteine peptide Cys-Cys-Pro-Gly-Cys-Cys (CCPGCC) with excellent 
(a)

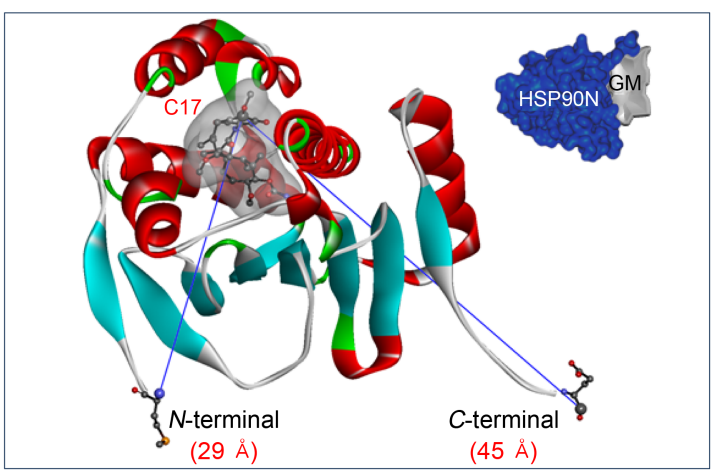

(b)
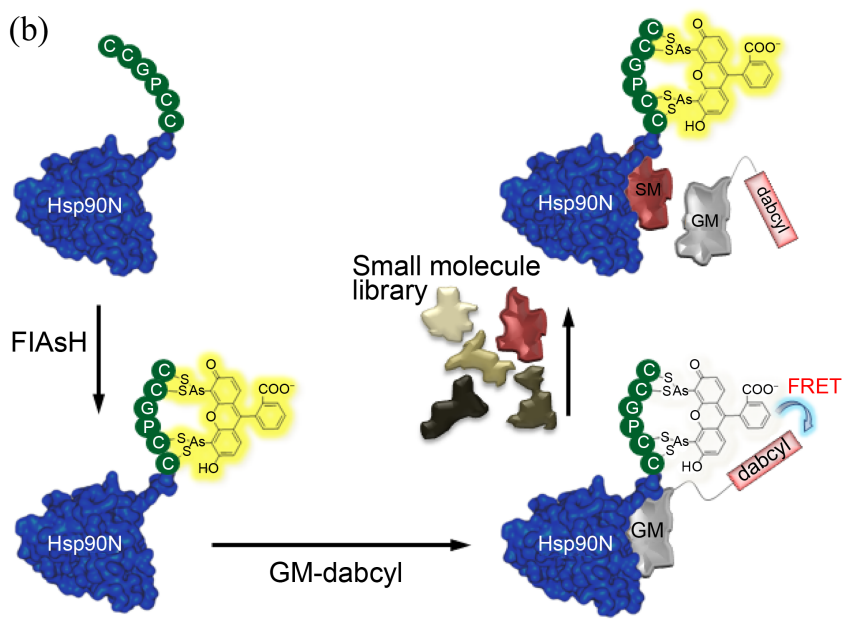

Figure 1. (a) Distances from GM to $N$ - or $C$-terminus in the crystal structure of GM-bound Hsp90N and (b) schematic diagram of the FRET-based assay system using FlAsH-labeled Hsp90N via the tetracysteine residue (GM, geldanamycin; SM, small molecule).

specificity and affinity. ${ }^{14}$ Because the biarsenical fluorescein derivative FlAsH-EDT 2 becomes strongly emissive only after it binds the tetracysteine motif, the site-specific fluorescent labeling of Hsp90N can be accomplished without tedious washings or protein purification steps to remove the excessive unlabeled fluorescent dye. On the basis of GMbound Hsp90N X-ray crystal structure, the distance from the GM C17 methoxy group to the Hsp90N $N$ - or $C$-terminus was 29 and $45 \AA$, respectively. These distances confirm that the $\mathrm{N}$ - and $\mathrm{C}$-termini of Hsp90N are within the FRET radius from the ATP-binding pocket (Figure 1(a)). For the selection of an appropriate FRET acceptor/quencher, we tested whether the dabcyl group was able to quench FlAsH emission. Excess dabcyl acid addition to the model FlAsH complex generated with FlAsH-EDT 2 and the synthetic tetracysteine peptide (WDCCPGCCK) led to a significant fluorescence emission reduction due to the proximal existence of the dabcyl group, as expected (Figure S1 in ESI $\dagger$ ). When the dabcyl moiety is conjugated to GM, the effective molarity of the dabcyl group is enhanced via the specific binding of GM-dabcyl at the ATP-binding pocket of the Hsp90 Nterminal domain (Figure 1(b)).

The crystal structure of GM-bound Hsp90N revealed that the $\mathrm{C} 17$ position of GM protrudes from the ATP-binding pocket of Hsp90N. In addition, the inhibitory activity of
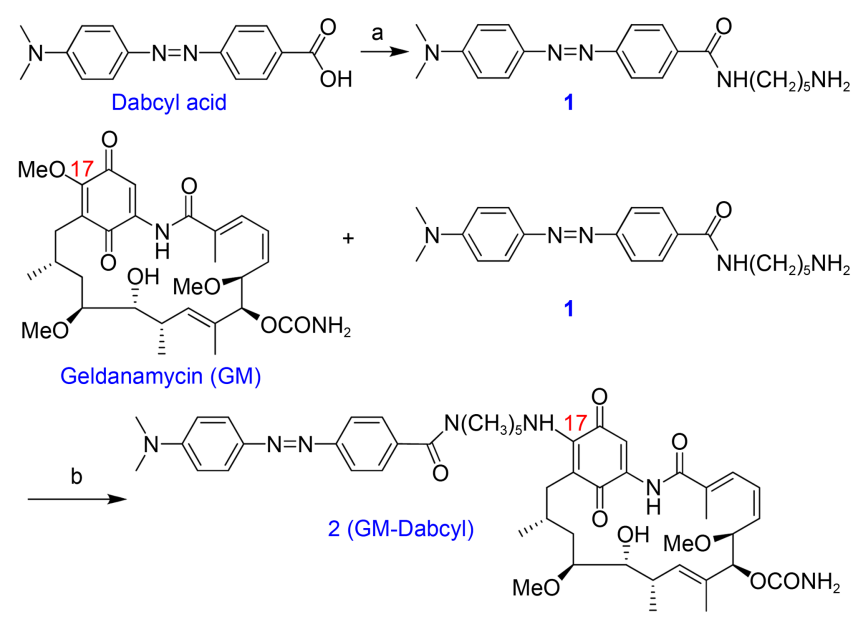

Scheme 1. Synthetic scheme of GM-dabcyl. Reagents and conditions: (a) dabcyl acid, 1,5-diaminopentane, EDC, HOBt, rt, 2 h, 96\%; (b) geldanamycin, compound 1, DIPEA, DMF, rt, 10 h, 95\%.

the GM derivatives against Hsp90 is tolerant only to its chemical modification at the $\mathrm{C} 17$ position. This structureactivity relationship supports the design of the GM-derived, clinical candidates 17-AAG and 17-DMAG, which possess substituent changes at the $\mathrm{C} 17$ position to improve their pharmacokinetic properties. ${ }^{8}$ For the preparation of the GMdabcyl FRET quencher, we introduced the dabcyl group via the amide coupling of dabcyl acid with 1,5-diaminopentane, followed by subsequent nucleophilic substitution at the $\mathrm{C} 17$ position of GM (Scheme 1).

To identify an effective FRET system, we designed and prepared two different FRET donor types through the sitespecific labeling of Hsp90N with FlAsH via protein engineering. As such, the tetracysteine motif was incorporated at the $N$ - or $C$-terminus of Hsp90N (Hsp90N-N and Hsp90N$\mathrm{C}$, respectively). The protein expressions of wild-type Hsp90N (Hsp90N-WT), Hsp90N-N, and Hsp90N-C were confirmed by SDS-PAGE and MALDI-TOF analyses (Figure 2(a) and Figure S2 in ESI + ). Unlike Hsp90N-WT, both Hsp90N-N and Hsp90N-C showed a significant fluorescence enhancement at $530 \mathrm{~nm}$ with an excitation at $508 \mathrm{~nm}$ upon treatment of FlAsH-EDT 2 . These signals confirm the specific labeling of FlAsH to the tetracysteine motif on Hsp90N. We also observed the expected FRET quenching after the addition of GM-dabcyl to FlAsH-labeled Hsp90N-N and Hsp90N-C (Figure 2(b)).

Considering that FRET is highly dependent on the distance between the donor and acceptor, the competitive binding of small molecules with GM-dabcyl leads to the deterioration of FRET quenching. As a proof-of-concept experiment, we induced the competitive binding of GMdabcyl with GM at various concentrations. As shown in Figure 2(c) and 2(d), GM can effectively inhibit the binding of GM-dabcyl with Hsp90N and the consequent reduction of FRET-induced fluorescence. Based on these experiments, we selected Hsp90N-C for the further HTS development because of its effective fluorescence enhancement upon specific FlAsH labeling, FRET-induced fluorescence reduc- 

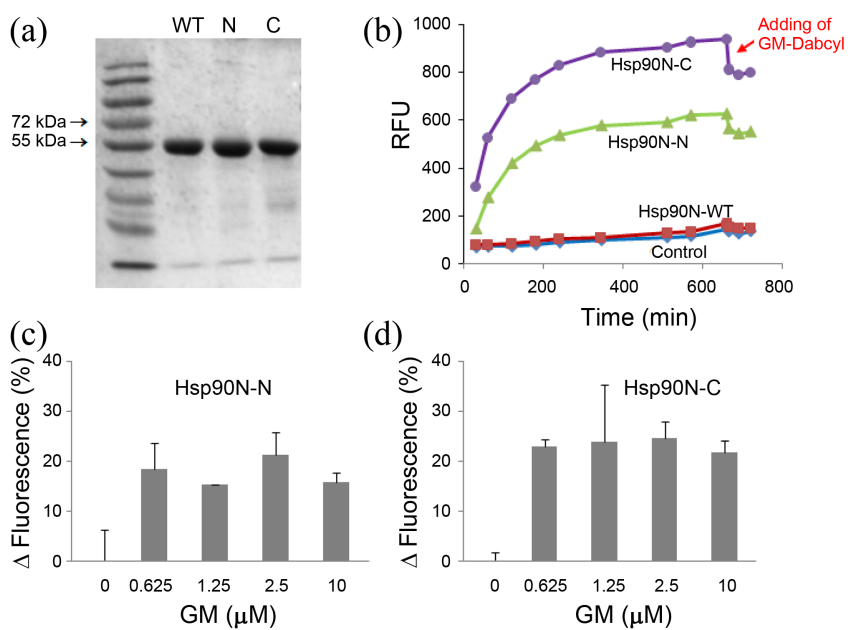

(d)

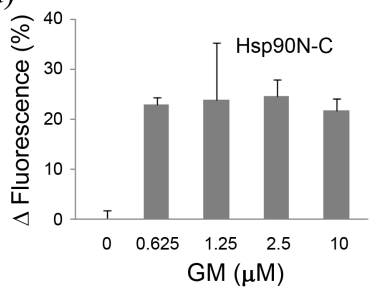

Figure 2. Inhibition of GM-dabcyl quenching by competitive binding of GM. (a) SDS-PAGE analyses of Hsp90N-WT, -N, and -C. (b) Fluorescence changes upon covalent labelling of FlAsH-EDT 2 on Hsp90N-WT, -N, and -C. (c, d) After incubation with serially diluted GM, $0.5 \mu \mathrm{M}$ of GM-dabcyl was added to the mixture, and the decreased fluorescence was measured for Hsp90N-N and Hsp90N-C, respectively.

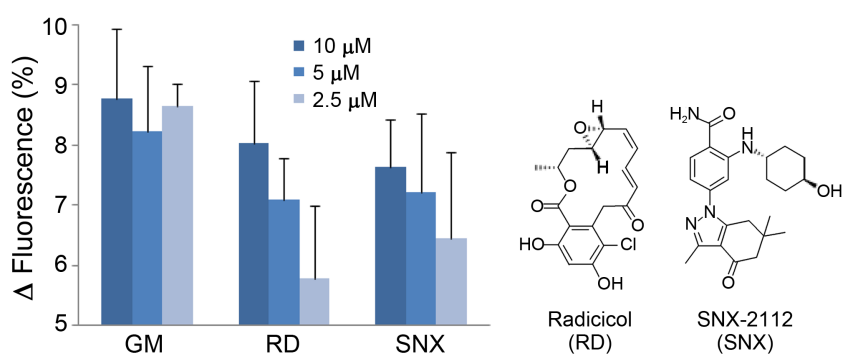

Figure 3. The competitive binding assay was performed with known Hsp90 inhibitors, GM, radicicol (RD) and SNX-2112 (SNX) at various concentrations in a 96-well plate format.

tion upon GM-dabcyl addition, and high fluorescence difference upon its competitive binding.

To practically implement this system in a high-throughput manner, we further optimized our FRET-based assay by screening three known Hsp90N inhibitors - GM, radicicol (RD), and SNX-2112 (SNX) - as positive controls in a 96well plate format. As shown in Figure 3, we observed the effective deterioration of FRET-induced fluorescence quenching by GM-dabcyl through the competitive binding of known Hsp90 inhibitors in a dose-dependent manner, even though this signal did not accurately reflect the binding affinity difference among the Hsp90N inhibitors. These data confirmed that FRET-based assay was robust and reliable for the primary screening of small-molecule Hsp90N inhibitors via competitive binding with GM at the ATP-binding pocket of $\mathrm{Hsp} 90 \mathrm{~N} N$-terminal domain.

With this FRET-based system in hand, we pursued the HTS of an in-house DOS library with diverse core skeletons ${ }^{15}$ using a 96-well plate format. The purified Hsp90N-C was first pre-incubated with FlAsH-EDT 2 for its specific labeling on tetracysteine moiety at the $C$-terminus of $H$ sp90N.

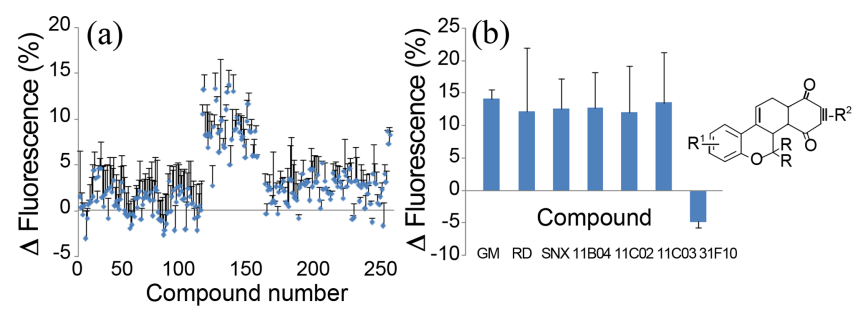

Figure 4. Representative data set of the FRET-based HTS assay for the discovery of small-molecule Hsp90N inhibitors. (a) Scatter plot showing the activities of 250 screened compounds from an inhouse DOS library. (b) Competitive binding confirmation of hit compounds using fluorescence spectrophotometry.

Without removal of unlabelled FlAsH-EDT 2 via washing or dialysis, the resulting FlAsH-labeled Hsp90N-C was dispensed into the individual wells of a 96-well plate charged with individual small molecules at an initial concentration of $10 \mu \mathrm{M}$. The fluorescence difference was measured before and after the addition of GM-dabcyl, which enabled the identification of competitive small-molecule binders of Hsp90N. DMSO was used as a negative control and known Hsp90N inhibitors, including GM, RD, and SNX as positive controls. The representative data of fluorescence change upon treatment of individual compounds is shown in Figure 4(a). To validate the FRET-based HTS results, we selected three primary hit compounds - 11B04, 11C02 and 11C03. In Figure 4(b), all three primary hit compounds with an identical core skeleton containing benzopyranyl substructure inhibited FRET-induced fluorescence quenching by more than $10 \%$, which was cross-confirmed by their competitive binding against GM-dabcyl at the ATP-binding pocket of Hsp90N$\mathrm{C}$ using fluorescence spectrophotometry. This reduction is comparable to the activity of known Hsp90 inhibitors. Hit compounds $11 \mathrm{~B} 04,11 \mathrm{C} 02$, and $11 \mathrm{C} 03$ were further evaluated for their anticancer activity using $\mathrm{T}$ lymphocyte Jurkat cells and showed relatively good inhibition of cell proliferation in dose-dependent manner (Figure S3 in ESI $\dagger$ ). These results are consistent with those of known Hsp90 inhibitors.

\section{Conclusion}

Although extensive researches have clearly demonstrated specific Hsp90 inhibitors as a promising approach for the development of novel therapeutic agents for various cancers, a limited number of assay systems exist with a proper platform and potential miniaturization for high-throughput evaluation. In this paper, we described the design and development of a FRET-based HTS system to identify competitive inhibitors of the ATP-binding pocket on the $N$ terminal domain of Hsp90. We utilized the spatial proximity of small-molecule inhibitor GM with protein receptor Hsp90N upon its specific binding and designed an assay system incorporating of a FRET donor and quencher. For our FRET-based system, we selected biarsenical fluorescein derivative FlAsH as a FRET donor because FlAsH can be specifically conjugated to Hsp90N via the introduction of a 
tetracysteine moiety either at its $C$ - or $N$-terminus through protein engineering. Since FlAsH becomes strongly emissive only after it binds to the tetracysteine motif, the site-specific fluorescent labeling of Hsp90N can be accomplished without tedious washing steps or further protein purification to remove the excessive fluorescent dye. For the FRET quencher, dabcyl moiety was conjugated at the $\mathrm{C} 17$ position of GM on the basis of X-ray crystal structure and structureactivity relationship studies. Using this FRET-based system, we pursued the preliminary HTS of our in-house DOS library for the discovery of novel competitive inhibitors of Hsp90N and identified a series of Hsp90 inhibitors with a new molecular framework. Further biological evaluation of these Hsp90 inhibitors will be reported in a future publication.

Supporting Information Available. Detailed synthetic procedures, biological procedures, characterization data and copies of NMR spectra for all compounds.

Acknowledgments. This study was supported by the National Research Foundation of Korea (NRF), MarineBio Technology Program funded by the Ministry of Land, Transport, and Maritime Affairs (MLTM), Korea, and the WCU program of the NRF funded by the Korean Ministry of Education, Science, and Technology (MEST). This study was also supported by AstraZeneca VRI research award program. S.O., Y.K., H.L., and J.K. were grateful for the fellowships awarded by the BK21 program.

\section{References}

1. (a) Young, J. C.; Moarefi, I.; Hartl, F. U. J. Cell Biol. 2001, 154, 267. (b) Whitesell, L.; Lindquist, S. L. Nat. Rev. Cancer 2005, 5, 761. (c) Sharp, S.; Workman, P. Adv. Cancer Res. 2006, 95, 323. (d) Neckers, L. J. Biosci. 2007, 32, 517.

2. (a) Chiosis, G.; Vilenchik, M.; Kim, J.; Solit, D. Drug Discov. Today 2004, 9, 881. (b) Calderwood, S. K.; Khaleque, M. A.;
Sawyer, D. B.; Ciocca, D. R. Trends Biochem. Sci. 2006, 31, 164 (c) Biamonte, M. A.; Van de Water, R.; Arndt, J. W.; Scannevin, R. H.; Perret, D.; Lee, W.-C. J. Med. Chem. 2010, 53, 3.

3. (a) Stebbins, C. E.; Russo, A. A.; Schneider, C.; Rosen, N.; Hartl, F. U.; Pavletich, N. P. Cell 1997, 89, 239. (b) Roe, S. M.; Prodromou, C.; O'Brien, R.; Ladbury, J. E.; Piper, P. W.; Pearl, L. H. J. Med. Chem. 1999, 42, 260. (c) Neckers, L.; Schulte, T. W.; Mimnaugh, E. Invest. New Drugs 1999, 17, 361.

4. (a) Kelland, L. R.; Sharp, S. Y.; Rogers, P. M.; Myers, T. G.; Workman, P. J. Natl. Cancer Inst. 1999, 91, 1940. (b) Banerji, U.; Walton, M.; Raynaud, F.; Grimshaw, R.; Kelland, L.; Valenti, M.; Judson, I.; Workman, P. Clin. Cancer Res. 2005, 11, 7023.

5. Solit, D. B.; Chiosis, G. Drug Discov. Today 2008, 13, 38.

6. (a) Chandarlapaty, S.; Sawai, A.; Ye, Q.; Scott, A.; Silinski, M.; Huang, K.; Fadden, P.; Partdrige, J.; Hall, S.; Steed, P.; Norton, L.; Rosen, N.; Solit, D. B. Clin. Cancer Res. 2008, 14, 240. (b) Okawa, Y.; Hideshima, T.; Steed, P.; Vallet, S.; Hall, S.; Huang, K.; Rice, J.; Barabasz, A.; Foley, B.; Ikeda, H.; Raje, N.; Kiziltepe, T.; Yasui, H.; Enatsu, S.; Anderson, K. C. Blood 2009, $113,846$.

7. Carreras, C. W.; Schirmer, A.; Zhong, Z.; Santi, D. V. Anal. Biochem. 2003, 317, 40.

8. Llauger-Bufi, L.; Felts, S. J.; Huezo, H.; Rosen, N.; Chiosis, G Bioorg. Med. Chem. Lett. 2003, 13, 3975.

9. Zhou, V.; Han, S.; Brinker, A.; Klock, H.; Caldwell, J.; Gu, X. Anal. Biochem. 2004, 331, 349.

10. Avila, C.; Hadden, M. K.; Ma, Z.; Kornilayev, B. A.; Ye, Q.-Z.; Blagg, B. S. J. Bioorg. Med. Chem. Lett. 2006, 16, 3005.

11. Galam, L.; Hadden, M. K.; Ma, Z.; Ye, Q.-Z.; Yun, B.-G.; Blagg, B. S. J.; Matts, R. L. Bioorg. Med. Chem. 2007, 15, 1939.

12. (a) Clegg, R. M. Curr. Opin. Biotechnol. 1995, 6, 103. (b) Selvin, P. R. Nat. Struct. Bio. 2000, 7, 730.

13. (a) Tsien, R. Y. Ann. Rev. Biochem. 1998, 67, 509. (b) LippincottSchwartz, J.; Patterson, G. H. Science 2003, 300, 87.

14. (a) Griffin, B. A.; Adams, S. R.; Tsien, R. Y. Science 1998, 281, 269. (b) Adams, S. R.; Campbell, R. E.; Gross, L. A.; Martin, B. R.; Walkup, G. K.; Yao, Y.; Llopis, J.; Tsien, R. Y. J. Am. Chem. Soc. 2002, 124, 6063.

15. (a) Ko, S. K.; Jang, H. J.; Kim, E.; Park, S. B. Chem. Commun 2006, 2962. (b) An, H.; Eum, S.-J.; Koh, M.; Lee, S. K.; Park, S. B. J. Org. Chem. 2008, 73, 1752. (c) Kim, Y.; Kim, J.; Park, S. B. Org. Lett. 2009, 11, 5214. (d) Oh, S.; Jang, H. J.; Ko, S. K.; Ko, Y.; Park, S. B. J. Comb. Chem. 2010, 12, 548. 